\title{
The Green function for simplest quantum graphs
}

\author{
K. K. Sabirov ${ }^{1}$ U. A. Aminov², Kh.Sh. Saparov², M. K. Karimov², Kh. Abdikarimov² \\ ${ }^{1}$ Physics Faculty, National University of Uzbekistan, Tashkent, Uzbekistan \\ ${ }^{2}$ Urganch State University, Urganch, Uzbekistan \\ karimjonsabirov@yahoo.com
}

PACS 03.65.Ge, 02.30.Ik

DOI 10.17586/2220-8054-2015-6-6-762-766

\begin{abstract}
We treat the problem of the Green function for quantum graphs by focusing on such topologies as star and tree graphs. The exact Green function for the Schrödinger equation on primary star graphs is derived in the form of $3 \times 3$ - matrix using the vertex boundary conditions providing continuity and current conservation. Extension of the approach for the derivation for the Green function on tree graph is presented. Possible practical applications of the obtained results are discussed.
\end{abstract}

Keywords: quantum graphs, Green function, vertex boundary conditions.

Received: 1 November 2015

\section{Introduction}

Particle and wave transport in branched systems has attracted much attention in different topics of physics such as optics, polymer and cold atom physics, condensed matter and biophysics. In most cases, such transport can be modeled by evolution equations on so-called metric graphs. The latter is called quantum graphs for nanometer scales and has become a rapidly developing subject over the last three decades (see, e.g., reviews [1-3] and references therein). In this case, one deals with the Schrödinger equation for which the boundary conditions on graph vertices graphs are imposed [1].

The simplest case for propagation of linear waves in branched optical structures is described by the Helmholtz equation on graphs. Formally, it coincides with the Schrödinger equation, which was extensively studied in the context of quantum chaos theory [1-10]. Linear waves in optical waveguide networks provide experimental realization of quantum graphs [11].

The Schrödinger equation on graphs was first introduced by Pavel Exner in $[5,6]$. Strict mathematical formulation of Schrödinger equation on graphs was given by Kostrykin and Schrader in [7], who formulated general boundary conditions, providing the self-adjointness of the problem. Nonrelativistic particle dynamics in networks described by the Schrödinger equation on graphs has been studied in the context of quantum chaos in the Refs. [1-3]. Despite the progress made in the study Schrödinger equation on graphs, the Green function based approach to particle transport still remains undeveloped. The only attempts for treatment of Green function on metric graphs are those in Refs. [12-14]. In [12] the Green function is constructed for the regular Shturm-Liouville problem on the interval via different approaches. The Green function for the Schrödinger operator on the open star graph is studied by the multiscattering expansion in [13]. Schmidt, Cheng and da Luz [14] presented a recursive prescription to calculate the exact Green function for general quantum graphs via a scattering approach. However, these works do not provide an explicit form of the Green function, even for star graph. 
In this paper, we address the problem of derivation of the exact Green function for the simplest quantum graphs in an explicit form using the Schrödinger equation with boundary conditions providing continuity and the current conservation at the graph's vertex. In quantum physics, the Green function is of importance for different problems, such as quantum transport, vacuum fluctuations, heat transport, and many others. In the case of quantum networks, knowledge of the Green function greatly facilitates transport phenomena in such systems and the Casimir effect in branched systems [15-19].

\section{Schrödinger equation on metric graphs}

Our task is to derive Green function for the Schrödinger equation on metric graphs. A graph represents system of bonds which are connected at one or more vertices (branching points). The connection rule is called the topology of a graph. When the bonds can be assigned a length, the graph is called a metric graph. The Schrödinger equation on a metric graph having $N$ represents system of $N$ - equations which are connected to each other via the vertex boundary conditions $[1,7]$. In the following we deal with the Schrödinger equation on the primary star graph (see 1) $\Gamma$ having three bonds with the lengths $L_{j}, j=1,2,3$ of finite bonds $b_{j}, j=1,2,3$ given as:

$$
-\frac{1}{2} \frac{d^{2}}{d x^{2}} \psi_{j}=E \psi_{j}
$$

The vertex boundary conditions for such graph can be written as:

$$
\begin{array}{r}
\psi_{1}(0)=\psi_{2}(0)=\psi_{3}(0), \\
\left.\frac{\partial}{\partial x} \psi_{1}\right|_{x=0}+\left.\frac{\partial}{\partial x} \psi_{2}\right|_{x=0}+\left.\frac{\partial}{\partial x} \psi_{3}\right|_{x=0}=0 \\
\psi_{j}\left(L_{j}\right)=0, j=1,2,3 .
\end{array}
$$

Eq.(1), together with the boundary conditions given by Eqs.(2)-(4), define the quantum

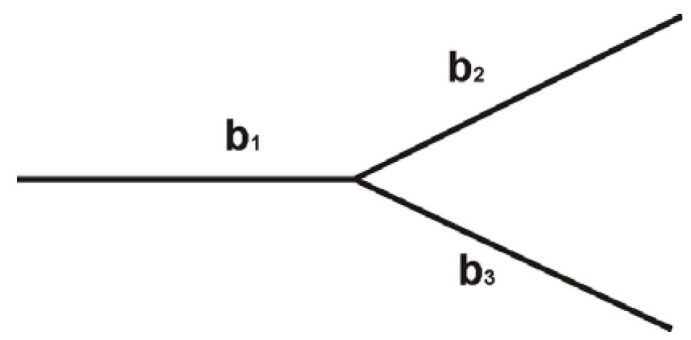

FIG. 1. Primary star graph consisting of 3 bonds connected at a vertex

mechanical problem of the quantum graph presented in Fig.1. Below, we will use Green function approach for this problem and derive explicit Green functions for this system.

\subsection{The Green function for quantum star graph}

The Green function for Schrödinger equation on star graph is defined as the solution of the following equation:

$$
-\frac{d^{2}}{d x^{2}} G\left(x, x^{\prime}\right)-2 E G\left(x, x^{\prime}\right)=2 \delta\left(x-x^{\prime}\right) I_{3},
$$


(where $I_{3}$ is $3 \times 3$ the identity matrix) with the boundary conditions:

$$
\begin{array}{r}
G_{1 j}\left(0, x^{\prime}\right)=G_{2 j}\left(0, x^{\prime}\right)=G_{3 j}\left(0, x^{\prime}\right), j=1,2,3, \\
\left.\frac{\partial}{\partial x} G_{1, j}\left(x, x^{\prime}\right)\right|_{x=0}+\left.\frac{\partial}{\partial x} G_{2, j}\left(x, x^{\prime}\right)\right|_{x=0}+\left.\frac{\partial}{\partial x} G_{3, j}\left(x, x^{\prime}\right)\right|_{x=0}=0, j=1,2,3, \\
G_{j, m}\left(L_{j}, x^{\prime}\right)=0, j, m=1,2,3, \\
\left.\frac{\partial}{\partial x} G_{j, m}\left(x, x^{\prime}\right)\right|_{x=x^{\prime}-0}-\left.\frac{\partial}{\partial x} G_{j, m}\left(x, x^{\prime}\right)\right|_{x=x^{\prime}+0}=-2 \delta_{j, m}, j, m=1,2,3 .
\end{array}
$$

Let us assume that equation:

$$
-\frac{1}{2} \frac{d^{2}}{d x^{2}} \varphi_{j}(x)-E \varphi_{j}(x)=f_{j}(x)
$$

obeys the boundary conditions given by Eqs.(2)-(4). Then the solution of Eq.(11) can be written as:

$$
\varphi_{j}(x)=\sum_{m=1}^{3} \int_{0}^{L_{m}} G_{j m}\left(x, x^{\prime}\right) f_{m}\left(x^{\prime}\right) d x^{\prime}
$$

It is clear that from the boundary conditions (6)-(8), one can obtain boundary conditions (2)-(4), i.e. the solution of Eq. (12) satisfies the boundary conditions given by Eqs. (2)-(4).

Thus, the Green function for a star graph presented in Fig.1 can be written as:

$$
G\left(x, x^{\prime}\right)=\frac{1}{i \sqrt{2 E}} e^{i \sqrt{2 E}\left|x-x^{\prime}\right|} I_{3}+\frac{1}{i \sqrt{2 E}} \sin (\sqrt{2 E} x) \cdot \mu^{(1)}\left(x^{\prime}\right)+\frac{1}{i \sqrt{2 E}} \cos (\sqrt{2 E} x) \cdot \mu^{(2)}\left(x^{\prime}\right),
$$

where $I_{3}$ is the $3 \times 3$ identity matrix, with $\mu^{(1)}, \mu^{(2)}$ are $3 \times 3$ are matrices, which obey the boundary conditions given by Eqs.(6)-(8):

$$
\begin{gathered}
\mu_{j m}^{(1)}=\left\{\begin{array}{c}
\frac{1}{\Delta}\left[i e^{i \sqrt{2 E} x^{\prime}} \Delta_{j}-e^{i \sqrt{2 E}\left(L_{j}-x^{\prime}\right)} \sum_{n \neq j} \Delta_{n, j}\right], m=j, \\
\frac{1}{\Delta}\left[i e^{i \sqrt{2 E} x^{\prime}} \Delta_{j}+e^{i \sqrt{2 E}\left(L_{m}-x^{\prime}\right)} \Delta_{j, m}\right], m \neq j,
\end{array}\right. \\
\mu_{j m}^{(2)}=-\frac{1}{\Delta}\left[i e^{i \sqrt{2 E} x^{\prime}} \prod_{n=1}^{3} \sin \left(\sqrt{2 E} L_{n}\right)+e^{i \sqrt{2 E}\left(L_{m}-x^{\prime}\right)} \prod_{n \neq m} \sin \left(\sqrt{2 E} L_{n}\right)\right],
\end{gathered}
$$

here $\Delta_{j}=\cos \left(\sqrt{2 E} L_{j}\right) \prod_{n \neq j} \sin \left(\sqrt{2 E} L_{n}\right), \Delta_{j, m}=\cos \left(\sqrt{2 E} L_{j}\right) \prod_{n \neq j, m} \sin \left(\sqrt{2 E} L_{n}\right), \Delta=\sum_{j=1}^{3} \Delta_{j}$, $j, m=1,2,3$.

This Green function can be used for solving of the transport phenomena and vacuum effect such as Casimir effect on branched structures and networks [15-19].

\section{Other simplest graph}

Here, we briefly show that the above approach can be used to derive the Green function for other simple graphs, such as the tree graph presented in Fig.2. In the case of 
the Schrödinger equation given by (1), one can impose the following boundary conditions:

$$
\begin{array}{r}
\psi_{1}(0)=0, \psi_{1 m n}\left(L_{1 m n}\right)=0, m, n=1,2, \\
\psi_{1}\left(L_{1}\right)=\psi_{1 m}\left(L_{1}\right), m=1,2, \\
\left.\frac{\partial}{\partial x} \psi_{1}\right|_{x=L_{1}}+\left.\sum_{m=1}^{2} \frac{\partial}{\partial x} \psi_{1 m}\right|_{x=L_{1}}=0, \\
\psi_{1 m}\left(L_{1 m}\right)=\psi_{1 m n}\left(L_{1 m}\right), m, n=1,2, \\
\left.\frac{\partial}{\partial x} \psi_{1 m}\right|_{x=L_{1 m}}+\left.\sum_{n=1}^{2} \frac{\partial}{\partial x} \psi_{1 m n}\right|_{x=L_{1 m}}=0 .
\end{array}
$$

The Green function for a tree graph having seven bonds, $G\left(x, x^{\prime}\right)$ is a $7 \times 7$-matrix obeying

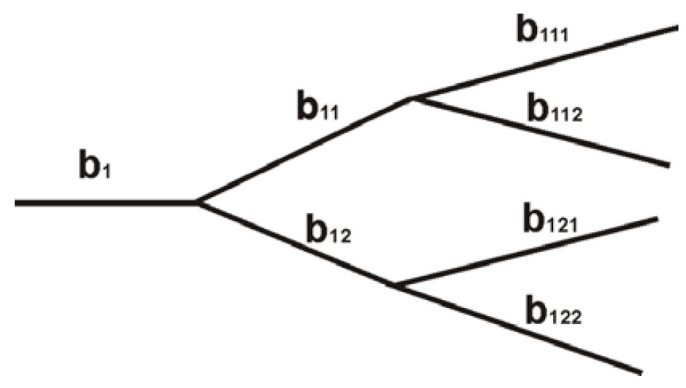

FIG. 2. Tree graph

the following conditions:

$$
\begin{array}{r}
G_{1, b}\left(0, x^{\prime}\right)=0, G_{1 m n, b}\left(L_{1 m n}, x^{\prime}\right)=0, m, n=1,2, \\
G_{1, b}\left(L_{1}, x^{\prime}\right)=G_{1 m, b}\left(L_{1}, x^{\prime}\right), m=1,2, \\
\left.\frac{\partial}{\partial x} G_{1, b}\left(x, x^{\prime}\right)\right|_{x=L_{1}}+\left.\sum_{m=1}^{2} \frac{\partial}{\partial x} G_{1 m, b}\left(x, x^{\prime}\right)\right|_{x=L_{1}}=0, \\
G_{1 m, b}\left(L_{1 m}, x^{\prime}\right)=G_{1 m n, b}\left(L_{1 m}, x^{\prime}\right), m, n=1,2, \\
\left.\frac{\partial}{\partial x} G_{1 m, b}\left(x, x^{\prime}\right)\right|_{x=L_{1 m}}+\left.\sum_{n=1}^{2} \frac{\partial}{\partial x} G_{1 m n, b}\left(x, x^{\prime}\right)\right|_{x=L_{1 m}}=0, m=1,2,
\end{array}
$$

$(b \in\{1 ; 11 ; 12 ; 111 ; 112 ; 121 ; 122\})$ and the Green function can be written as:

$$
G\left(x, x^{\prime}\right)=\frac{1}{i \sqrt{2 E}} e^{i \sqrt{2 E}\left|x-x^{\prime}\right|} I_{7}+\frac{1}{i \sqrt{2 E}} \sin (\sqrt{2 E} x) \cdot \mu^{(1)}\left(x^{\prime}\right)+\frac{1}{i \sqrt{2 E}} \cos (\sqrt{2 E} x) \cdot \mu^{(2)}\left(x^{\prime}\right),
$$

where $I_{7}-7 \times 7$ is the unit matrix, $\mu^{(1)}, \mu^{(2)}$ are $7 \times 7$ - matrices, which obey the boundary conditions given by Eqs.(19)-(23). It is clear that a similar way can be used to obtain the Green function of a tree graph with arbitrary number of bonds as well as other simplest topologies.

\section{Conclusion}

In this paper, using the direct approach based on the definition of the Green function, we derived the explicit Green functions for the simplest graphs such as star and tree graphs. 
The approach can be used for the derivation of the Green function of other simple topologies. The above results can be useful for the study of particle transport in quantum networks such as polymers, molecular chains, lattices and other discrete structures. An important problem where knowledge of the Green function on graphs is important is the problem of the Casimir effect on networks [15-19]. Using of the Green function approach for calculating the zero point energy is an effective tool, and in many cases, allows one to avoid divergencies [20].

\section{References}

[1] T. Kottos and U. Smilansky. Periodic orbit theory and spectral statistics for quantum graphs, Ann.Phys., 1999, 76, P. 274.

[2] S. Gnutzmann, U. Smilansky. Quantum graphs: Applications to quantum chaos and universal spectral statistics, Adv.Phys., 2006, 55, P. 527.

[3] S. GnutzmannJ.P., Keating b, F. Piotet. Eigenfunction statistics on quantum graphs, Ann.Phys., 2010, 325, P. 2595.

[4] L. Pauling. The Diamagnetic Anisotropy of Aromatic Molecules, J. Chem. Phys., 1936, 4, P. 673.

[5] P. Exner, P. Seba, P. Stovicek. Quantum interference on graphs controlled by an external electric field. J. Phys. A: Math. Gen., 1988, 21, P. 4009-4019.

[6] P. Exner, P. Seba. Free quantum motion on a branching graph, Rep. Math. Phys., 1989, 28, P. 7.

[7] R. Kostrykin, R. Schrader. Kirchhoff's rule for quantum wires, J. Phys. A: Math. Gen., 1999, 32, P. 595.

[8] J. Boman, P. Kurasov. Symmetries of quantum graphs and the inverse scattering problem, Adv. Appl. Math., 2005, 35, P. 58.

[9] T. Cheon, P. Exner, O. Turek. Approximation of a general singular vertex coupling in quantum graphs, Ann.Phys., 2010, 325, P. 548.

[10] J.P. Keating. Fluctuation statistics for quantum star graphs. Quantum graphs and their applications, Contemp. Math., 2006, 415, P. 191.

[11] O. Hul et al. Experimental simulation of quantum graphs by microwave networks, Phys. Rev. E, 2004, 69, P. 056205.

[12] W. J. Parnell. Greens functions, integral equations and applications, MATH 34032, Spring 2013.

[13] F. Barra, P. Gaspard. Transport and dynamics on open quantum graphs, Physical review E, 2001, 65, P. 016205.

[14] A. G. M. Schmidt, B. K. Cheng, M.G.E. da Luz. Green function approach for general quantum graphs, J. Phys. A: Math. Gen., 2003, 36, P. L545L551.

[15] P. Calabrese, M. Mintchev, E. Vicari. Entanglement entropy of quantum wire junctions, J. Phys. A: Math. Theor., 2012, 45, P. 105206.

[16] B. Bellazzini, M. Burrello, M. Mintchev, P. Sorba. Quantum field theory on star graphs, Proc. Symp. Pure Math., 2008, 77, P. 639.

[17] B. Bellazzini, M. Mintchev. Quantum fields on star graphs, J. Phys. A, 2006, 39, P. 11101.

[18] B. Bellazzini, M. Mintchev, P. Sorba. Quantum fields on star graphs with bound states at the vertex, J. Math. Phys., 2010, 51, P. 032302.

[19] S. A. Fulling, L. Kaplan, J. H. Wilson. Vacuum energy and repulsive Casimir forces in quantum star graphs. Physical review A, 2007, 76, P. 012118.

[20] G. Plunien, B. Muller, W. Greiner, The Casimir Effect, Phys.Rep., 1986, 134, P. 87. 